\title{
Creating Spectral Templates from Multicolor Redshift Surveys
}

\author{
T. Budavári ${ }^{1}$, A.S. Szalay, A.J. Connolly ${ }^{2}$, I. Csabai ${ }^{1}$, M.E. Dickinson ${ }^{3}$ \\ Department of Physics and Astronomy, The Johns Hopkins University, \\ Baltimore, MD 21218 \\ and the HDF/NICMOS Team
}

\begin{abstract}
We present a novel method capable of creating optimal eigenspectra from multicolor redshift surveys for photometric redshift estimation. Our iterative training algorithm modifies the templates to represent the photometric measurements better. We present a short description of our algorithm here. We show that the corrected templates give more precise photometric redshifts, essentially a "free" feature, since we were not fitting for the redshifts themselves.
\end{abstract}

\section{Introduction}

In the last couple of years photometric redshift estimation has become a very powerful tool in getting statistical information about our universe and also frequently used for spectroscopic target selection to measure spectra of faint galaxies. Different techniques have been developed since Baum ('62) but all of them may be classified into two groups based on the approach they use.

The first are the so called empirical methods (Connolly et al. '95a). Having a set of objects with spectroscopic and photometric data, one can easily obtain an analytic, usually polynomial fitting function for the redshift over the color "hyperplane". Lower order piecewise fits can be also applied efficiently (Brunner et al. "99). The function can be very quickly evaluated at any photometric data point. However, this method has some disadvantages. We need to have a very good training set to get the fitting formula and we still will not be able to extrapolate far from the training set. The fitting formula only works for a specific set of passbands and limited redshift ranges, thus if we want to get photometric redshifts for different catalogs, we need training sets for all the photometric standards, so it is very hard to get consistent redshift estimations for them.

In the template or SED (spectral energy distribution) fitting methods a set of restframe spectra are used to work out what spectrum and redshift give better

\footnotetext{
${ }^{1}$ Department of Physics, Eötvös Loránd University, Budapest, H-1088

${ }^{2}$ Department of Physics and Astronomy, University of Pittsburgh, Pittsburgh, PA 15260

${ }^{3}$ Space Telescope Science Institute, Baltimore, MD 21218
} 
representation of the color of a galaxy. Using this approach gives us not just the redshift of the galaxy but also an approximate spectral type information. The technique works very well on separate photometric catalogs with different filter sets but it is obvious that the basis spectra are crucial. The currently used template spectra come from both synthetic (Bruzual \& Charlot '93) and measured (Coleman, Wu \& Weedman '80) spectral libraries but generally they are applied as they come with the hope that they are good enough. An advanced version of the template fitting algorithm has been developed to use "continuum number" of spectra using eigenspectra instead of a large number of actual spectra in a very efficient way in terms of CPU time and memory. Using eigenspectra (Connolly et al. '95b) means we consider all spectra to be approximately a linear combination of a small number of spectra, the eigenspectra. It also turns out there is an optimal subspace filtering method (see AJC's talk; Budavári et al. '99) which gives more reliable restframe spectra than direct coefficient fitting.

In this paper we describe a new method which has been developed to bring together the advantages of both techniques. We show how to train eigenspectra in an iterative procedure to represent the photometry better. In the training procedure different catalogs and also measured spectra can be incorporated. The resulting eigentemplates can be used for redshift estimation on any photometry catalog and the redshift prediction becomes more accurate.

\section{Template Reconstruction}

Our goal is to find out the underlying basis template spectra that can be used later for photometric redshift estimation. Since we observe galaxies at different redshifts, their restframe spectra are sampled at different wavelengths by the blueshifted filters. If we have a deep enough multicolor redshift survey (or more) then the rough SEDs defined by the blueshifted passbands are overlapping with each other. This means the eigenspectra are oversampled and this is the fact which allows us to derive applications for extracting high resolution eigentemplates from broadband photometry (Csabai et al. '99). Our new approach is different mainly in spectrum repairation that we introduce in the next section.

The iterative training procedure follows a fully statistical approach. Its robustness is provided by the Karhunen-Loève (KL) expansion (Karhunen '47; Loève '48). This transformation has been used to derive eigentemplates from spectra and this is just what we do in our iterative training. Starting from a set of eigenspectra we can compute a best fitting type for each and every galaxy in our photometric catalog based on the known redshift. This gives us an approximation to the restframe spectra of the galaxies. Now we can check how good the spectrum is at least at such wavelengths where photometric data are available. We modify - repair — "smoothly" the spectra to represent the measured values better. This spectrum correction is the only tricky step that we will describe later on. Having all the modified spectra we can invoke the KL transformation and obtain a new set of eigentemplates. The iteration can be continued until the correlation between simulated and measured flux values is strong enough. 

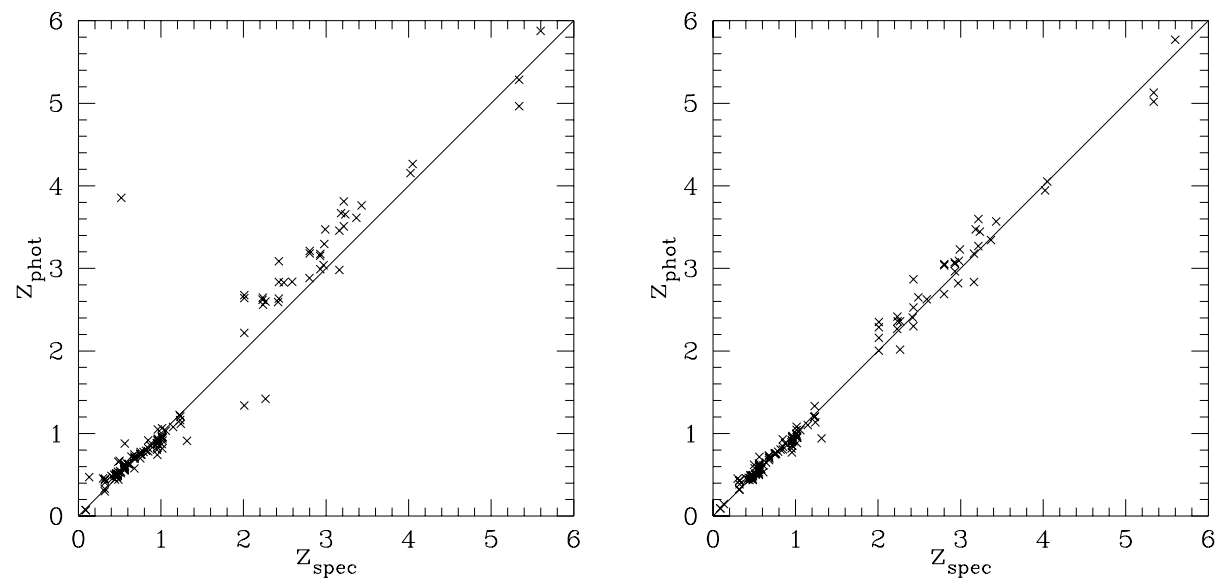

Figure 1. Correlation between spectroscopic and photometric redshifts. On the right - using $3 \mathrm{CWW}$ eigenspectra, left - using $3 \mathrm{KL}$ eigenspectra after 30 iterations. See Figure 3. for corresponding eigenspectra.

Repairing Spectra. The way we modify the spectrum of a galaxy is the key step of our training algorithm. The best fitting linear combination of the eigenspectra is the current approximation of the galaxy that we are about to correct due to the photometric values. This is a multidimensional minimization problem for the spectrum itself, where the dimension of the problem is determined by the spectral resolution. The cost function is built up by two terms. The first part corresponds to the deviation of the spectrum based on simulated fluxes from the measured photometric data and the second part is the deviation of the spectrum from the template based linear combination.

$$
\chi^{2}(\vec{s})=\sum_{n} \frac{1}{\Delta_{n}^{2}}\left\{f_{n}-\hat{f}_{n}(\vec{s})\right\}^{2}+\sum_{k} \frac{1}{\sigma_{k}^{2}}\left\{s_{k}-s_{k}^{t}\right\}^{2}
$$

$\vec{s}$ is the discrete representation of the spectrum and $s_{k}$ is the $k$ th element of $\vec{s}$, where $k$ refers to the wavelength, $\lambda_{k} . s_{k}^{t}$ stands for the template based spectrum and $\sigma_{k}$ describes the ability of the spectrum to be changed at a given wavelength. The photometric error in the $n$th passband is represented by $\Delta_{n}$. This minimization problem can be reduced to a system of linear equations, since $\hat{f}_{n}(\vec{s})$ is linear in its variables, $\left\{s_{k}\right\}$; so it is easy to solve. The new KL basis built from the modified spectra will span a different subspace which represents the photometry better. The details of this method will be described elsewhere (Budavári et al. '99).

\section{Application}

Our training algorithm has been applied to the HDF/NICMOS catalog (Williams et al. '96; Dickinson et al. '99) which has unique photometric data quality and there is a reasonable number of objects with spectroscopic redshift (Cohen, '98). 

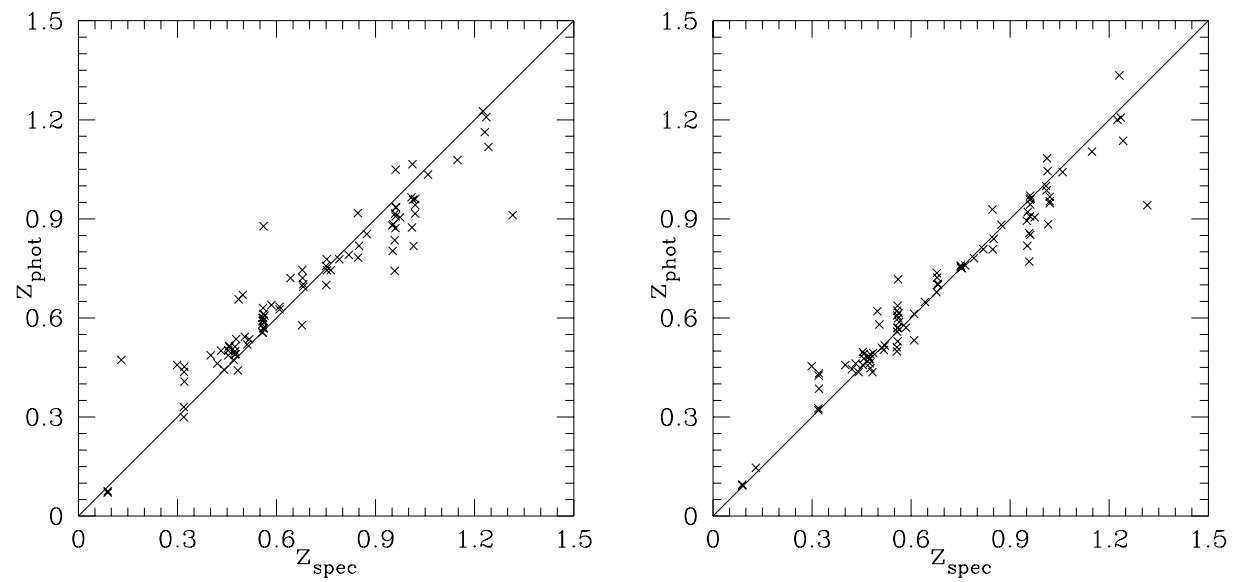

Figure 2. Redshift limited scatter plot showing a close-up of Figure 11.

There are photometric data available in seven passbands: 4 HDF filters (F300W, F450W, F606W, F814W), 2 NICMOS filters (F110W, F160W) and a groundbased K'. Our initial eigentemplates were derived from the Coleman, Wu \& Weedman (CWW) spectra that usually provide better redshift estimation than others (Fernandez-Soto et al., '99; Hogg et al., '98).

After a few iterations the redshift scatter becomes tighter while the eigentemplates just slightly change. The comparison between the original and resulting eigenspectra after 30 iterations (KL-30 ) can be seen on Figure 3. This tiny difference between them was enough to improve the redshift scatter plot by a factor of two as it can be seen on Figure 11. and 2. The corresponding statistical errors were calculated for two redshift ranges. The overall statistics and a redshift limited sample were evaluated. Beyond the standard root mean square error $\left(\Delta_{\text {rms }}\right)$ the relative deviation of $(1+z)$ was also computed $\left(\Delta_{\text {rel }}\right)$ for comparison with estimates found by other authors. Table 1. contains the calculated error estimates for both the CWW and KL-30 cases.

Table 1. Comparison of the errors in the fits based on the Coleman, Wu \& Weedman and the KL (after 30 iterations) eigenspectra

\begin{tabular}{cccc} 
Error & Range & CWW & KL-30 \\
\hline \hline$\Delta_{\text {rms }}$ & $z<6$ & 0.23 & 0.12 \\
$\Delta_{\text {rel }}$ & $z<6$ & 0.079 & 0.042 \\
\hline$\Delta_{\text {rms }}$ & $z<.8$ & 0.087 & 0.063 \\
$\Delta_{\text {rel }}$ & $z<.8$ & 0.050 & 0.035 \\
\hline
\end{tabular}

In order to test the robustness of the method we started the iteration from arbitrary constant functions, as well. After just a couple of iterations the $4000 \AA$ break was visible and other important features emerged soon from scratch. 


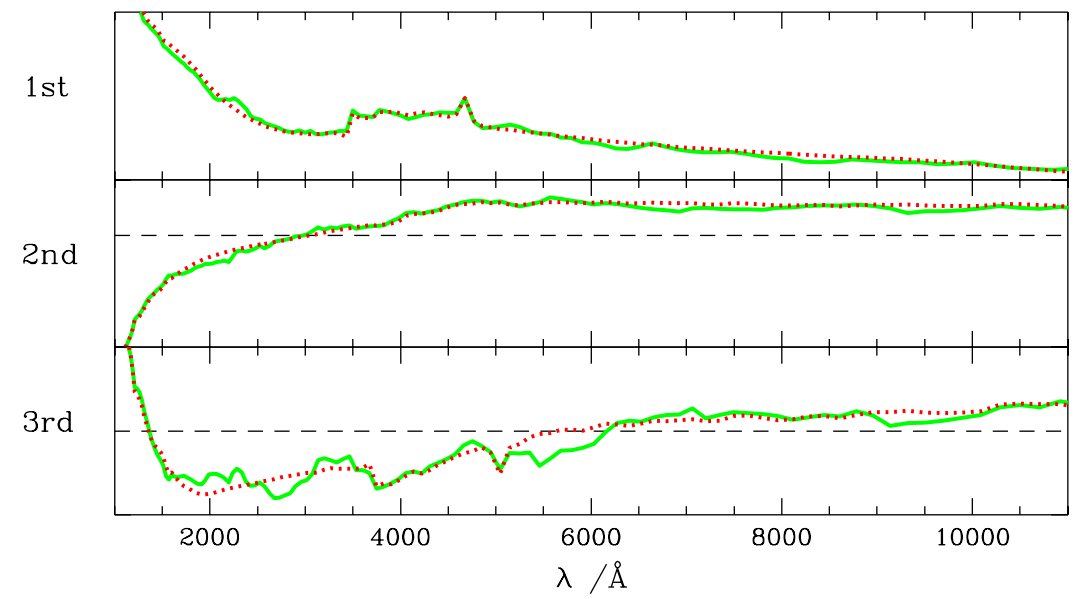

Figure 3. These figures show the initial eigenspectra derived from the Coleman, $\mathrm{Wu} \&$ Weedman spectra (dotted line) and the corrected KL spectra after 30 iterations (solid line). The horizontal dashed line signals the zero level. See Figure 11. and 2. for related redshift scatter plots.

The limitation on our current application is the relatively small number of galaxies with accurate multicolor photometry and redshifts in the Hubble Deep Field. Consequently the third eigenspectrum appears to be being over fit (we run out of degrees of freedom). As our technique is constructed to be applied to any set of multicolor observations we can incorporate many multicolor datasets from different sources (i.e. we do not need to train the relation for a given set of filters or magnitude limits). With the new generation of multicolor photometric and spectroscopic surveys nearing completion we, therefore, expect the accuracy of the derived spectral energy distributions to improve dramatically in the near future.

\section{Conclusions}

We have shown that the current templates used in SED fitting photometric redshift estimation can be modified to give better correlation between the actual photometry and the template based simulated fluxes. We presented a robust method that creates better eigenspectra in an iterative way and converges very quickly. The training procedure is very generic. Different catalogs can be incorporated at the same time even with different filtersets. It is also easy to extend the method to involve measured spectra if needed.

We showed that the new modified eigentemplates give us a better basis for photometric redshift estimation, even though the training procedure is not fitting for the redshift, in fact the photometric redshift estimation is only performed for monitoring purposes. 
Acknowledgments. IC and TB acknowledges partial support from the MTA-NSF grant no. 124 and the Hungarian National Scientific Research Foundation (OTKA) grant no. T030836, AS from NASA LTSA (NAG53503), HST Grant (GO-07817-04-96A), MED from HST (GO-07817-01-96A) and AJC from HST (GO-07817-02-96A) and LTSA (NRA-98-03-LTSA-039).

\section{References}

Baum, W.A., 1962, IAU Symposium No. 15, 390

Budavári, T., Szalay, A.S., Connolly, A.J., Csabai, I. \& Dickinson, M.E., 1999, in preparation, to be submitted to AJ

Brunner, R.J., Connolly, A.J., Szalay, A.S., 1999, ApJ, 516:563-581

Bruzual, A.G. \& Charlot, S., 1993, ApJ, 405, 538

Cohen, J.G., 1998, HDF Symposium (STScI symp. ser. 11), p. 52

Coleman, G.D., Wu., C.-C. \& Weedman, D.W., 1980, ApJS, 43, 393

Connolly, A.J., Csabai, I., Szalay, A.S., Koo, D.C., Kron, R.G. \& Munn, J.A., 1995a, AJ 110, 2655

Connolly A.J., Szalay A.S., Bershady M.A., Kinney A.L. \& Calzetti D., 1995b, AJ, 110, 1071

Csabai, I., Connolly, A.J., Szalay, A.S. \& Budavári, T., 1999, in press, submitted to AJ

Dickinson, M., 1999, in "After the Dark Ages: When Galaxies Were Young," eds. S. Holt and E. Smith, (AIP: Woodbury NY), p. 122

Dickinson et al., 1999, in preparation

Fernández-Soto, A., Lanzetta, K.M. \& Yahil, A., 1999, ApJ, 513:34-50

Hogg et al., 1998, ApJ, 499, 555

Karhunen, H., 1947, Ann. Acad. Science Fenn, Ser. A.I. 37

Loève, M., 1948, Processus Stochastiques et Mouvement Brownien, Hermann, Paris, France

Williams, R.E. et al., 1996, AJ, 112, 1335 